Biol. Cybern. 58, 287-294 (1988)

\title{
Properties of Individual Movement Detectors as Derived from Behavioural Experiments on the Visual System of the Fly
}

\author{
W. Reichardt and M. Egelhaaf \\ Max-Planck-Institut für biologische Kybernetik, Spemannstrasse 38, D-7400 Tübingen, Federal Republic of Germany
}

\begin{abstract}
The performance of the fly's movement detection system is analysed using the visually induced yaw torque generated during tethered flight as a behavioural indicator. In earlier studies usually large parts of the visual field were exposed to the movement stimuli; the fly's response, therefore, represented the spatially pooled output signals of a large number of local movement detectors. Here we examined the responses of individual movement detectors. The stimulus pattern was presented to the fly via small vertical slits, thus, nearly avoiding spatial integration of local movement information along the horizontal axis of the eye. The stimulus consisted of a vertically oriented sine-wave grating which was moved with a constant velocity either clockwise or counterclockwise. In agreement with the theory of movement detectors of the correlation type, the time-course of the detector signal is modulated with the spatial phase of the stimulus pattern. It can even assume negative values for some time during the response cycle and thus signal the wrong direction of motion. By spatially integrating the response over sufficiently large arrays of movement detectors these response modulations disappear. Finally, one obtains a signal of the movement detection system which is constant while the pattern moves in one direction and only changes its sign when the pattern reverses its direction of motion. Spatial integration thus represents a simple means to obtain a meaningful representations of motion information.
\end{abstract}

\section{Introduction}

It is now more than 30 years since the movement detector of the so-called correlation-type has been proposed to explain motion perception in insects (Hassenstein and Reichardt 1956; Reichardt 1957, 1961; Reichardt and Varjú 1959; Varjú 1959). In the meantime, good evidence has been accumulated that this motion detection scheme can also be applied to motion detection in humans (e.g. van Doorn and Koenderink 1982a, b; van Santen and Sperling 1984; Wilson 1985; Baker and Braddick 1985). The mechanism underlying motion detection is non-linear and local. Roughly speaking, movement detection is based on the multiplication-like interaction of the appropriately filtered signals of neighbouring retinal input channels. During the last years our interest in the basic mechanism underlying movement detection has focused on dynamical aspects and the dependence of the detector output on the structure of the stimulus pattern as well as the properties of twodimensional arrays of movement detectors (Reichardt and Guo 1986; Egelhaaf and Reichardt 1987). In the present paper we concentrate on the functional properties of individual movement detectors and the significance of spatial integration in representing meaningful information on a moving pattern. Theoretical predictions based on the movement detector theory are compared with corresponding experimental results obtained by evaluating the visually induced yaw torque of flying flies as a convenient indicator for movement perception.

\section{Materials and Methods}

The behavioural experiments were carried out with wild type female house-flies Musca domestica (L.) which were obtained from laboratory cultures. The flies were prepared as described by Fermi and Reichardt (1963). The head of the testflies was fixed to the thorax by a mixture of wax and colophonium; thereby the ocelli were carefully coated. A small triangular piece of cardboard was fixed to the frontal part of the animal's thorax. With this triangle the flies were suspended from a torque compensator which prevented both rotatory and translatory movements of the animal and allowed direct measurement of the in- 
stantaneous yaw torque generated by the fly (e.g. Fermi and Reichardt 1963). The signals were stored by a signal averager (Princeton, 4202), transfered to a computer (Apple IIc) and further processed.

The animals were positioned in the centre of a stationary white cylinder with a diameter and height of $70 \mathrm{~mm}$ and $57 \mathrm{~mm}$, respectively. The cylinder had two rectangular openings ("slits"); their height amounted to $62^{\circ}$ as seen by the fly, their horizontal angular extent to either $8^{\circ}, 20^{\circ}$, or $40^{\circ}$, respectively. The slits were located symmetrically in front of both eyes, with their centre at $\pm 40^{\circ}$ with respect to the fly's frontal midline. The stimulus panorama could be moved around the cylinder. As a consequence, the fly was only exposed to it via the slits. The stimulus pattern consisted of a vertical sinusoidal grating with a spatial wavelength of $40^{\circ}$ and a contrast $\left(I_{\max }-I_{\min }\right) /\left(I_{\max }+I_{\min }\right)$ of 0.16 . It was illuminated from behind by three direct current driven fluorescent ring bulbs. By means of a specially arranged reflection cone the cylinder surrounding the fly was homogeneously illuminated from above.

The computer simulations were carried out on an IBM-AT using the ASYST-software (Macmillan Software Company, Keithley Instruments).

\section{Functional Representation of a Moving Pattern at the Output of a Movement Detector}

A detector of the type as proposed to underly motion detection in insects (Hassenstein and Reichardt 1956; Reichardt 1957, 1961; Reichardt and Varjú 1959; Varjú 1959) is displayed in Fig. 1 in its most simplified form. It has two input channels which are spatially separated by a small interval $\Delta x$. The movement detector is composed of two subunits that are mirror images of each other. These subunits share two input channels that sample the visual field at two neighbouring points in space. $F(x, t)$ represents a onedimensional spatio-temporal luminance pattern, i.e. the luminance varies as a function of the spatial location $x$ and time $t$. The input signals of a detector, therefore, are $F(x, t)$ and $F(x+\Delta x, t)$, respectively. The signal of one branch of each subunit is assumed to pass through a linear temporal filter. For simplicity, this filter will be approximated by a pure delay $\varepsilon$. Computer simulations have shown (see Fig. 2) that this does not affect the principle conclusions of this paper. In each subunit of the detector the delayed signal coming from one input channel is multiplied with the instantaneous signal of the neighbouring input channel. The final detector output is given by the difference between the two subunit outputs

$$
\begin{aligned}
D(x, t)= & F(x, t-\varepsilon) \cdot F(x+\Delta x, t) \\
& -F(x+\Delta x, t-\varepsilon) \cdot F(x, t) .
\end{aligned}
$$

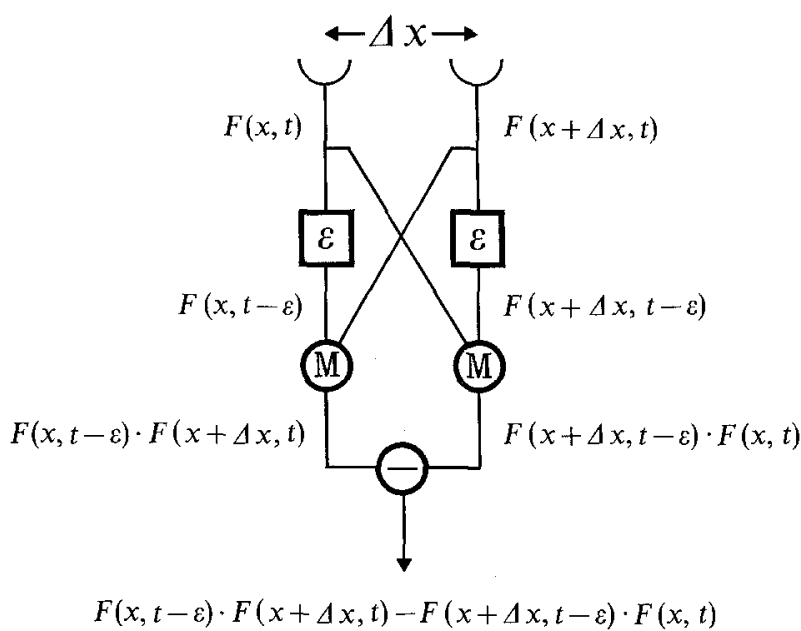

Fig. 1. Schematic representation of a movement detector and its formal operations. A movement detector consists of two mirrorsymmetrical subunits. In the simplest version of the model the input signal of one branch of each subunit is delayed by a brief time interval $\varepsilon$. In each subunit the delayed signal originating from one retinal location is multiplied with the undelayed signal of the neighbouring input channel. The final detector output is given by the difference of the responses of the two subunits. $F(x, t)$ represents the input signal and $\Delta x$ the spatial distance of the two movement detector input stages

The output of a single movement detector to an almost arbitrary moving pattern can be represented in a first approximation by the following functional expression

$D(x, t) \approx-\varepsilon \cdot \mathrm{d} s(t) / \mathrm{d} t \cdot\left[(\partial F / \partial x)^{2}-F \cdot \partial^{2} F / \partial x^{2}\right]$

which has been derived previously (Reichardt and Guo 1986) and further elaborated in subsequent studies (Egelhaaf and Reichardt 1987; Reichardt 1987). $\mathrm{d} s(t) / \mathrm{d} t$ represents the instantaneous pattern velocity, where $s(t)$ is the time-dependent spatial displacement of the pattern. It should be noted that the theoretical approach which led to (2) is based on the assumption that the spatial separation between the detector input channels is infinitesimally small. Roughly speaking, this means that it can only be applied if the spatial wavelength of the stimulus pattern is much larger than the inter-receptor distance. This is, however, not a too restrictive condition for most practical purposes (see Egelhaaf and Reichardt 1987). This condition is met by the stimulus pattern used in our experiments (see below).

From (2) it is obvious that a movement detector of the type discussed here is not a pure velocity sensor. Instead, its output is the product of both the stimulus velocity $\mathrm{d} s(t) / \mathrm{d} t$ and a term which depends in a complicated non-linear way on the time-dependent detector input function. On the basis of this formalism it has been predicted (Reichardt and Guo 1986; 

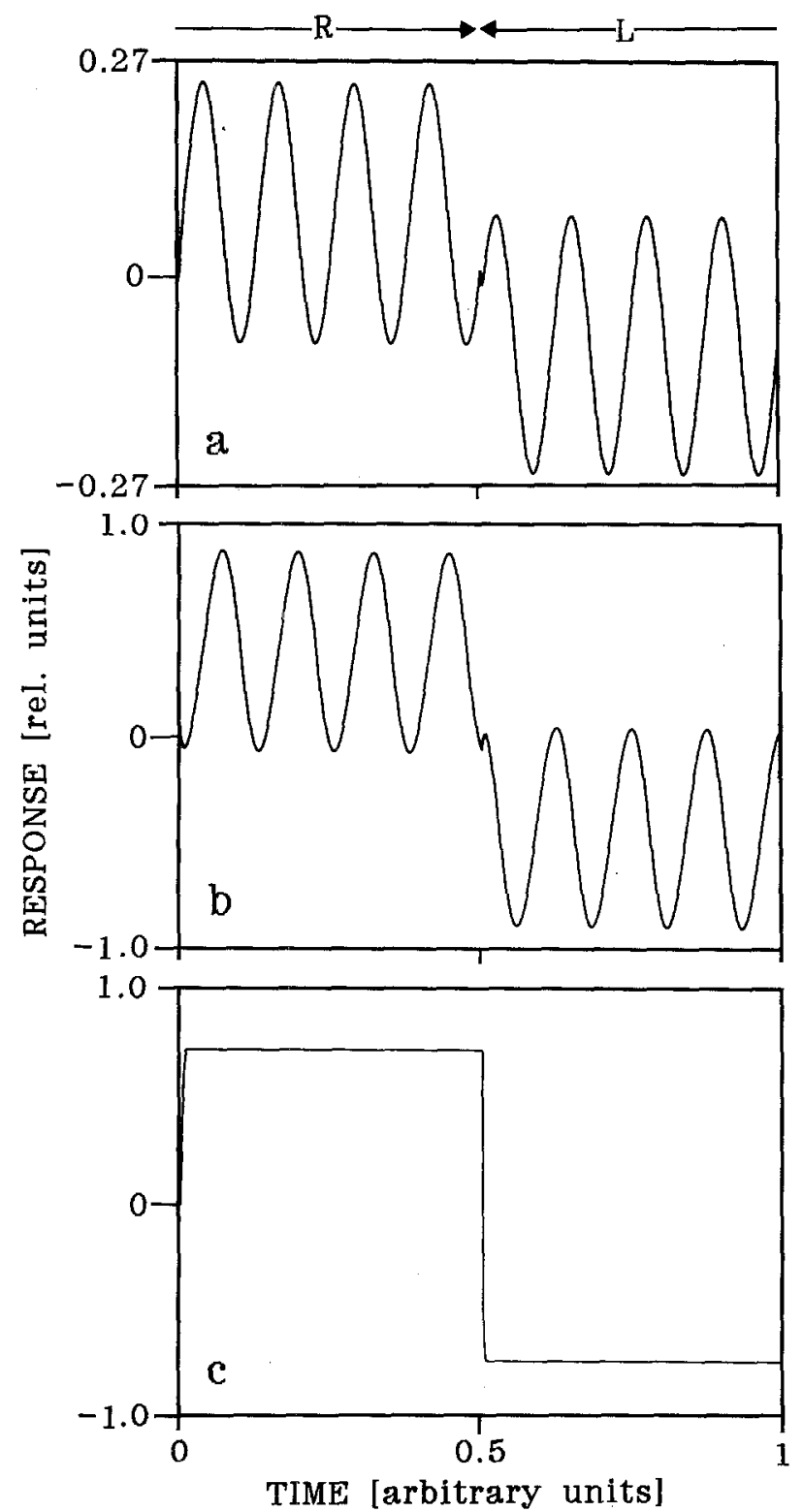

Fig. 2a-c. Computer simulation of a single movement detector and one-dimensional arrays of movement detectors. The stimulus pattern consisted of a sine-wave grating which was first moved to the right and then to the left as is indicated at the upper margin of the diagram. One spatial period of the stimulus pattern is covered by 36 equidistant movement detectors. The spatial distance between the two input stages of a detector amounted to $1 / 36$ of the spatial wavelength. In a the response of only a single movement detector is shown. In $\mathbf{b}$ and $\mathbf{c}$ there is spatial integration over 18 and 36 detectors which cover $1 / 2$ of the spatial period or an entire wavelength of the pattern, respectively. In the simulations the modulation amounted to 0.5 . Instead of a pure delay, a first-order low-pass was used as movement detector filter in the computer simulations. The instantaneous response of a single detector is sinusoidally modulated and may even signal motion in the wrong direction. When there is spatial integration over increasingly larger detector arrays, the mean response level gradually increases, while the relative amplitude of the modulations decreases. The modulations finally disappear, when the spatial integration extends over an integer multiple of the spatial wavelength
Reichardt 1987) that the instantaneous response of a single movement detector, under certain stimulus conditions, can signal the wrong direction of motion, at least for some time. Provided that the stimulus pattern passes the movement detector with a constant velocity this can only happen, if the time-dependent expression in the brackets in (2) reverses its sign. [This reversal of the sign of the movement detector response should not be confused with a reversal in sign which can be observed, if the spatial wavelength of the stimulus pattern satisfies the relation $2 /(2 n+2) \cdot \Delta \varphi<\lambda$ $<2 /(2 n+1) \cdot \Delta \varphi$, with $n=0,1,2, \ldots$; this so-called "geometrical interference" is solely due to the finite distance between the retinal sampling stages (Varjú 1959; Götz 1964).] If, for instance, an edge is moved across a detector, the response signalling the proper direction of motion is preceeded or succeeded, depending on the polarity of the edge, by a response component in the wrong direction (see Reichardt and Guo 1986).

This characteristic property of the movement detector will be further analysed and experimentally challenged in the present study. This will be done here by using a one-dimensional sine-wave grating with a wavelength $\lambda$, instead of an edge, moving with a constant velocity $\mathrm{ds} / \mathrm{dt}=-c$. With $F(x, t)=I$ $+\Delta I \sin [2 \pi / \lambda \cdot(x-c t)]$ as detector input function one obtains from (2) for the movement detector output

$D(x, t) \approx \varepsilon c \cdot(2 \pi / \lambda)^{2} \Delta I[\Delta I+I \sin 2 \pi / \lambda \cdot(x-c t)]$,

where $I$ and $\Delta I$ designate the mean intensity and modulation, respectively. From this expression it is immediately obvious that the time-dependent movement detector response to a sine-wave grating moving with a constant velocity is sinusoidally modulated. The instantaneous detector response, however, is not always positive. Depending on the pattern contrast it can assume negative values for some time during the response cycle. A single movement detector then signals the wrong direction of motion. This is illustrated by the computer simulations shown in Fig. 2a; a sine-wave grating first moves to the right, while in the middle of the stimulation cycle it reverses its direction of motion. Although the mean responses during motion from left to right and right to left are positive and negative, respectively, the response profiles cross the zero line. It follows from (3) that only for a pattern contrast of $\Delta I / I$ of $100 \%$ the response profiles do not cross the zero line. Spatial integration of movement information over part of the visual field leads to an increasing mean response, while the relative amplitude of the modulations decreases. This is shown in Fig. $2 b$ where spatial integration extends over $1 / 2$ spatial wavelength of the stimulus pattern. The modulations completely vanish, if one integrates over an 
array of movement detectors which jointly cover with their receptive fields an integer number of spatial wavelengths of the pattern (see Fig. 2c). This can be derived from (3) where in this case the time-dependent expression disappears.

Finally it should be noted that it is an intrinsic property of movement detectors of the correlation type that they are selective for the direction of motion; irrespective of whether an individual movement detector or an integrated array of detectors is concerned, they mediate the same response profiles to motion in opposite directions, but with an inverted sign (see Fig. 2).

\section{Experimental Results}

In our experiments the optomotor yaw torque response was used as an indicator for the performance of the movement detection system. Since the optomotor response is driven by the spatially pooled output of large retinotopic arrays of horizontally oriented local movement detectors covering the entire visual field, the response of a single detector can only be studied if spatial integration is prevented in some way. Ideally it would be desirable to stimulate only the two input channels of an individual movement detector. Although this is, in principle, possible (see Kirschfeld 1972; Riehle and Franceschini 1984), this technique has serious disadvantages, as will be discussed below. Most importantly, if only moderate contrasts are used, the responses to single-detector stimulation are only small and require considerable averaging to be discriminable from the background noise. This problem can be overcome, if instead of only a single detector a large number of movement detectors is stimulated synchronously. In a first approximation, this can be achieved by exposing the eye to the stimulus pattern only via a vertical slit. If the pattern changes only along its horizontal extent and the slit is relatively small as compared to the spatial wavelength of the pattern, the corresponding input channels of different movement detectors which are distributed along the vertical extent of the slit virtually get the same input signals. A larger pattern wavelength, thus, allows one to use a wider slit and, consequently, to get a larger number of almost synchronously stimulated movement detectors involved in the behavioural response. On the other hand, if the spatial wavelength of the stimulus pattern is too large, the phase difference between the two input channels of a single movement detector and, consequently, its response will become too small. The slit width of $1 / 5$ spatial wavelength chosen in our behavioural experiments (see Fig. 3a) was found to be a good compromise in this respect.

Since the properties of movement detectors were intended to be analysed, stimulus conditions had to be

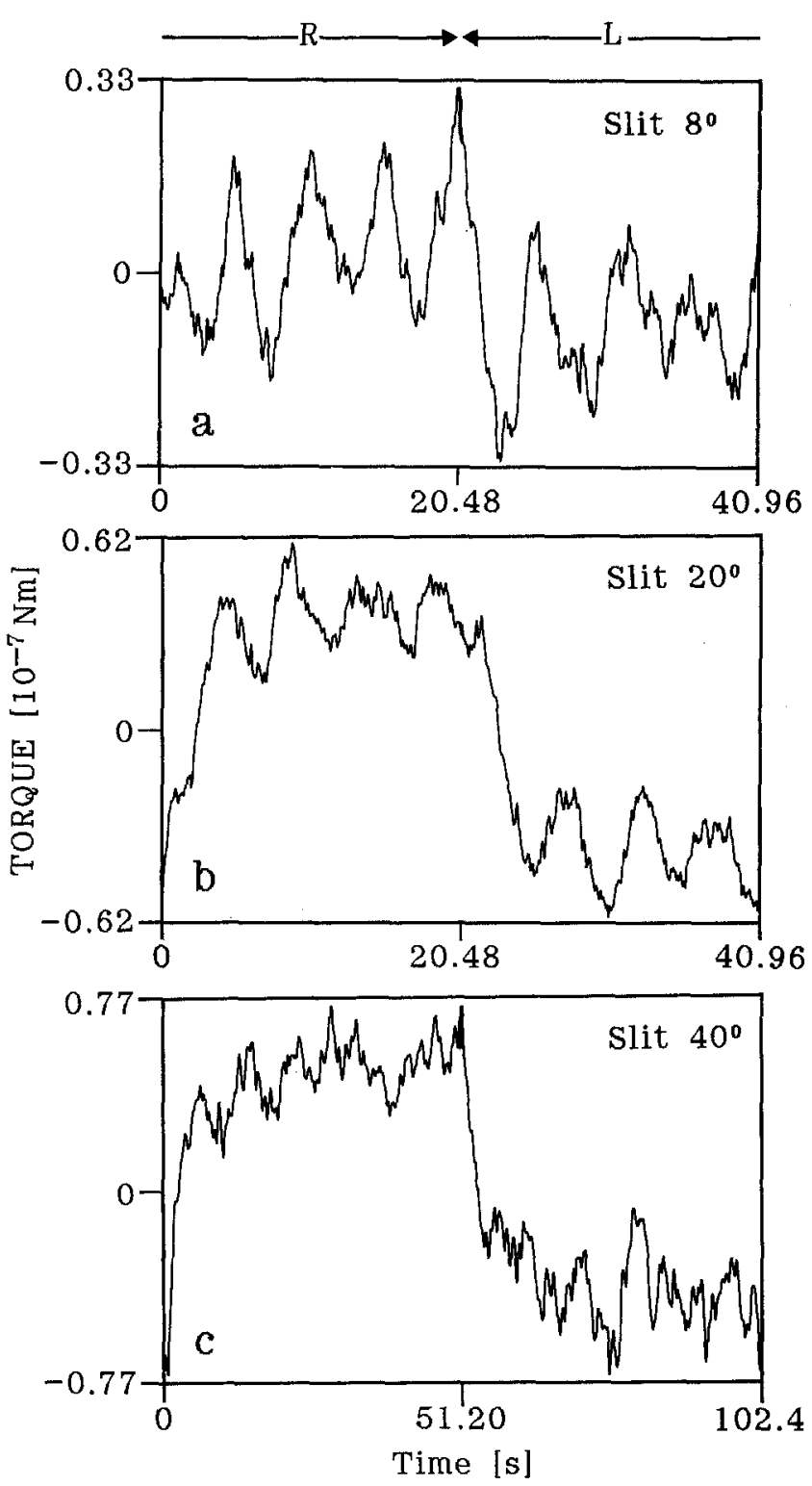

Fig. 3a-c. The consequences of spatial integration on the motion-induced optomotor yaw torque. The stimulus pattern consisted of a one-dimensional sine-wave grating with a spatial wavelength of $40^{\circ}$ and a contrast of 0.16 . The pattern was first moved to the right and then to the left as is indicated at the upper margin of the figure. The angular velocity of the stimulus pattern amounted to $7.81 \% \mathrm{~s}(\mathbf{a}, \mathbf{b})$ and $3.125 \% \mathrm{~s}(\mathbf{c})$, respectively. The data are averages from 8 flies, each stimulated 10 times with the entire stimulus programme. The test-fly was exposed to the stimulus pattern via two symmetrically placed vertical slits; their mean position was at $\pm 40^{\circ}$ as measured from the frontal mid-line of the animal. The width of the slits amounted to $8^{\circ}$ in a, $20^{\circ}$ in b, and $40^{\circ}$ in $\mathrm{c}$. This allowed the stimulation of different numbers of movement detectors. Consequently, spatial integration of movement information extends over parts of the visual field with a different size. When there is only little spatial integration (a), the instantaneous movement detector response is approximately sinusoidally modulated and may even cross the zero line. With a slit of $\frac{1}{2}$ spatial wavelength width (b) the modulations are still present but do not reach the zero line. They almost disappear, when the detector response is integrated over an integer multiple of the spatial wavelength of the stimulus pattern (c) 
found which allowed the movement detector response to be expressed in the optomotor yaw torque without being affected by processes others than the actual motion detection. This imposed several constraints on the choice of the stimulus conditions used in our behavioural experiments. First, patterns with a relatively low contrast $(0.16)$ were chosen; at higher contrasts non-linearities, such as saturation phenomena in the input channels get involved (Egelhaaf and Borst in preparation). Second, problems might arise from a kind of low-pass filter somewhere between the spatial integration of the motion detectors in the third visual ganglion of the fly's brain and the motor output (Egelhaaf 1987). To prevent this filter from modifying the time course of the movement detector output the contrast frequency of the stimulus had to be chosen to be sufficiently small, even if this meant sub-optimal stimulation of the movement detectors. Third, instead of only one slit two slits symmetrically arranged in front of the two eyes were used, while the stimulus pattern was alternately moved clockwise or counterclockwise. This was reasonable, since there is evidence that the yaw torque response is not only controlled by directionally selective motion detectors; there are also response components which are independent of the direction of motion and, thus, are directed towards the position of the stimulus (Pick 1976; see also Poggio and Reichardt 1976). When using two slits, the latter response components can be assumed to cancel each other.

Figure $3 \mathrm{a}$ illustrates the movement detector response as it manifests itself in our slit paradigm in the optomotor yaw torque. The fly was exposed to a cyclical stimulation programme. At first the pattern was moved clockwise with a constant velocity; then it reversed its direction and moved counter-clockwise in the second half of the stimulation cycle. For reasons of symmetry, this allows to determine the fly's zero level of torque response. Two features of the response profiles should be noted. $i$ ) The movement detector response to a sine-wave grating moving with a constant velocity is, in a first approximation, sinusoidally modulated as is predicted by the movement detector theory. This temporal modulation reflects the contrast frequency of the stimulus pattern. ii) The response profiles to clockwise and counter-clockwise motion cross the zero line, although the mean responses are positive and negative, respectively. This, however, means quite in accordance with the model predictions that the instantaneous output of a single movement detector can assume values which signal motion in the wrong direction.

These characteristics of the movement detector response disappear, if there is some sort of spatialintegration over larger arrays of movement detectors. It should be noted that there is good evidence that in the optomotor system of the fly the movement detectors are not integrated linearly but in a highly non-linear way (Reichardt et al. 1983). This, however, does not affect the conclusions to be drawn here. The effect of physiological spatial integration is illustrated in Fig. $3 \mathrm{~b}$ and c. With a slit of $1 / 2$ spatial wavelength width the sinusoidal modulations of the response are still pronounced but never reach the zero line. When the movement detector response is integrated over even larger detector arrays, the contrast frequency dependent temporal modulations of the response almost disappear. Only the more or less constant response plateaus syndirectional with the direction of motion are seen. This is what has to be expected, if over an integral number of spatial wavelengths is integrated. The residual small sinusoidal modulations of the response profile are due to the sensitivity gradients of the optomotor response within the visual field (e.g. Wehrhahn 1986).

\section{Discussion}

The time course of the response of movement detectors of the correlation-type has been studied theoretically and experimentally using the visually induced yaw torque of the fly as a behavioural indicator. As has been shown before, the visual system of the house-fly is a convenient model system for studying various visual information processing tasks and, in particular, the principle mechanisms underlying the evaluation of motion from the visual surround (e.g. Reichardt 1986, 1987). In the present study it has been concluded that the instantaneous response of a single movement detector - even to a stimulus pattern moving with a constant velocity in only one direction - does not directly signal the correct motion. Instead, it is modulated by the spatial phase of the stimulus pattern and may even signal motion in the wrong direction. It is particularly obvious from a recent theoretical formulation of the movement detector response (Reichardt and Guo 1986; Egelhaaf and Reichardt 1987; Reichardt 1987), which has been used here for the model predictions, that spatial integration over sufficiently large areas of the visual field is a simple means for these temporal modulations of the response to disappear. This prediction turned out to be correct for the motion detection system of the fly as is found in the behavioural measurements of the present study as well as in electrophysiological experiments on large-field motion sensitive neurones in the third visual ganglion (Egelhaaf, unpublished results).

\section{Alternative Approaches to Study the Properties of Individual Movement Detectors}

The most direct approach to studying the response of individual movement detectors would be to electrophysiologically record from those neurones in the 
brain which represent the local motion detectors. Although there is cursory evidence for local neurones in the fly's visual system which might play a role in the motion detection process (Mimura 1972; DeVoe and Ockleford 1976; DeVoe 1980), these cells are relatively small and hard to record from long enough to allow a systematic analysis of their properties. It is, however, interesting to note that the response of some of these units to a moving sine-wave grating is modulated in a way as expected from the detector theory (DeVoe 1980). This holds also true for certain types of directionally-selective motion-sensitive neurones in the vertebrate visual cortex (e.g. Holub and MortonGibson 1981). Whether these neurones represent the local motion detectors is not quite clear, and the experimental evidence in this respect is still confusing.

Other studies analysed the local interactions underlying motion detection in a more indirect way. As pioneered by Barlow and Levick (1965) in the rabbit retina spatially integrating units instead of the local movement detectors were recorded from, while it was tried to excite or inhibit single motion detectors with local stimuli simulating apparent motion. In this way spatial integration is avoided and the properties of single movement detectors can, at least in principle, be studied. Due to the ommatidial structure of the insect eye, it is possible to stimulate single photoreceptors individually. This fortunate situation has been exploited to study motion detection by recording from a large-field neurone (Riehle and Franceschini 1984). The results of this study are as is qualitatively predicted by the movement detector model discussed here, and thus represent a very specific evidence in favour of it.

The present study pursues still another strategy to determine the properties of individual movement detectors. Here the fly could see a one-dimensional stimulus pattern only through a vertical slit, which thus almost prevented spatial integration along the horizontal axis of the eye. We preferred this technique to single receptor stimulation, since it allowed us to use less extreme stimulus conditions. In particular, we used light adapted animals and moderate stimulus contrasts. This turned out to be necessary, since otherwise non-linearities such as saturation phenomena severely alter the time-course of the motion detector response (Egelhaaf unpublished; Egelhaaf and Borst, in preparation). Moreover, the characteristics of our stimuli were chosen so that the movement detector response could be treated analytically, and thus allowed us to make precise predictions which, subsequently, could be tested in the experiments.

\section{Significance of Spatial Integration}

Are the phase-dependent modulations of the movement detector output a characteristic feature of the particular motion detection scheme studied here? In principle, they can be also found in alternative formulations of the motion detector model, if no special measures are taken (see Adelson and Bergen 1985; van Santen and Sperling 1985). All these models, however, are equivalent to a movement detector of the correlation-type, if this detector is elaborated by appropriate spatio-temporal filters in its input stages (see van Santen and Sperling 1985). It is true that these "elaborations" affect the specific spatio-temporal transfer characteristic of the specific model implementation, but are not an essential precondition for the movement detector to work properly. Spatial bandpass filters in the input channels of the movement detectors represent one of these auxiliary assumptions. On the basis of neurophysiological (e.g. Baker and Cynader 1986; Emerson et al. 1987) and psychophysical (e.g. Pantle et al. 1978; Anderson and Burr 1985) evidence this appears to be adequate, if one is mainly concerned with motion perception in higher vertebrates and man. For the present purpose it is only important to note that this type of filtering virtually eliminates the mean luminance from the input signal. Of course, this is also the case if there is some kind of temporal high-pass filtering in the input channels. From (3) it is immediately clear that completely eliminating the mean luminance from the detector input eliminates the time-dependent modulation in its response to a pattern moving with a constant speed in one direction. The instantaneous output of the individual movement detector, thus, directly signals the correct motion and no spatial integration is required in this case. This is, however, only true as long as there are no non-linearities in the input channels which affect the mean value of the signal. Otherwise, the phasedependent modulations of the movement detector response are almost unavoidable.

Probably the simplest means to get rid of these modulations is either temporal or spatial integration over a sufficiently large retinotopic patch of movement detectors. In case of a periodic stimulus pattern this means that integration has to extend at least over one cycle of either the largest temporal or spatial frequency component in the input signal. The minimum range of integration, thus, depends on the spatial frequency content and velocity of the moving pattern as well as the properties of the spatio-temporal filters in the input channels of the movement detector. This implies that there is some kind of trade-off between the temporal and spatial detail which can be represented by the system evaluating movement information.

Some sort of spatial integration is quite common in cells which mediate information on motion in both vertebrates (e.g. Zeki 1974; Felleman and Kaas 1984) and invertebrates (e.g. Hausen 1984). In the latter class of cells it appears that their temporal properties are essentially determined by the time constants of the 
movement detectors in their input circuitry and that no significant temporal integration takes place (e.g. Egelhaaf and Reichardt 1987). In man the importance of spatial integration for the evaluation of motion information has been demonstrated in a number of psychophysical studies (e.g. Lappin and Bell 1976; Chang and Julesz 1983). Temporal integration, on the other hand, has also been shown to play a decisive role in certain motion evaluation tasks. This has been shown, for instance, for the landing system of the house-fly (Borst and Bahde 1986), in the occular following system of monkeys (e.g. Miles et al. 1986), and for man in various psychophysical studies (e.g. Burr 1981; van Doorn and Koenderink 1984). Particularly interesting in the present context is the study of van Doorn and Koenderink (1984) which revealed that the detectability of coherent motion is gouverned by a trade-off between the pattern size and the presentation time. It should be emphasized that the terms "spatial" and "temporal integration" are used here only in a colloquial and not in a mathematical sense. For instance, some form of non-linear spatial integration of local motion information has been analysed experimentally and theoretically in large-field neurones in the fly visual system which play a decisive role in the discrimination of objects from their background. These non-linearities can be explained on the basis of a non-linear transformation of the local movement detector output prior to spatial summation (Reichardt et al. 1983; Egelhaaf 1985a,b). The characteristic properties of spatial integration in the different vertebrate movement sensitive systems are less clear and need to be worked out experimentally and theoretically.

It should be noted, however, that these considerations are only concerned with one dimension of the two-dimensional array of movement detectors subserving the eye and, consequently, with onedimensional stimulus patterns only. In case of a twodimensional movement detector array the situation is much more complex. Even a separate representation of the movement detectors aligned along the horizontal and vertical axis of the eye, respectively, usually does not allow to immediately infer from the local motion measurements the correct direction of motion of the stimulus pattern (Reichardt 1987). To achieve this goal further information processing stages are certainly required. Some sort of spatial and/or temporal integration appears to be a simple means to obtain a meaningful representation of motion information by eliminating the phase-dependent modulations from the local motion measurements.

Acknowledgements. We are grateful to Dr. A. Borst for critically reading the manuscript, to $L$. Heimburger for drawing the figures and to I. Geiss for secretarial assistance.

\section{References}

Adelson EH, Bergen JR (1985) Spatiotemporal energy models for the perception of motion. J Opt Soc Am A 2:284-299

Anderson SJ, Burr DC (1985) Spatial and temporal selectivity of the human motion detection system. Vision Res 8:1147-1154

Baker LB, Braddick OJ (1985) Temporal properties of the shortrange process in apparent motion. Perception 14:181-192

Baker CL, Cynader MS (1986) Spatial receptive-field properties of direction-selective neurons in cat striate cortex. J Neurophysiol 55:1136-1152

Barlow HB, Levick WR (1965) The mechanism of directionally selective units in rabbit's retina. J Physiol 178:477-504

Borst A, Bahde S (1986) What kind of movement detector is triggering the landing response of the housefly? Biol Cybern 55:59-69

Burr DC (1981) Temporal summation of moving images by the human visual system. Proc R Soc Lond B 211:321-339

Chang JJ, Julesz B (1983) Displacement limits, directional anisotropy and direction versus form discrimination in random-dot cinematograms. Vision Res 23:639-646

DeVoe RD (1980) Movement sensitivities of cells in the fly's medulla. J Comp Physiol B 8:93-119

DeVoe RD, Ockleford EM (1976) Intracellular responses from cells of the medulla of the fly, Calliphora erythrocephala. Biol Cybern 23:13-24

van Doorn AJ, Koenderink JJ (1982a) Temporal properties of the visual detectability of moving spatial white noise. Exp Brain Res 45:179-188

van Doorn AJ, Koenderink JJ (1982b) Spatial properties of the visual detectability of moving white noise. Exp Brain Res 45:189-195

van Doorn AJ, Koenderink JJ (1984) Spatiotemporal integration in the detection of coherent motion. Vision Res $24: 47-53$

Egelhaaf M (1985a) On the neuronal basis of figure-ground discrimination by relative motion in the visual system of the fly. I. Behavioural constraints imposed on the neuronal network and the role of the optomotor system. Biol Cybern $52: 123-140$

Egelhaaf $\mathrm{M}(1985 \mathrm{~b})$ On the neuronal basis of figure-ground discrimination by relative motion in the visual system of the fly. III. Possible input circuitries and behavioural significance of the FD-cells. Biol Cybern 52:267-280

Egelhaaf M (1987) Dynamic properties of two control systems underlying visually guided turning in house-flies. J Comp Physiol A 161:777-783

Egelhaaf M, Reichardt W (1987) Dynamic response properties of movement detectors: theoretical analysis and electrophysiological investigation in the visual system of the fly. Biol Cybern 56:69-87

Emerson RC, Citron MC, Vaughn WJ, Klein SA (1987) Nonlinear directionally selective subunits in complex cells of cat striate cortex. J Neurophysiol 58:33-65

Felleman DJ, Kaas JH (1984) Receptive-field properties of neurons in middle temporal visual area (MT) of owl monkeys. J Neurophysiol 52:488-513

Fermi G, Reichardt W (1963) Optomotorische Reaktionen der Fliege Musca domestica. Abhängigkeit der Reaktion von der Wellenlänge, der Geschwindigkeit, dem Kontrast und der mittleren Leuchtdichte bewegter periodischer Muster. Kybernetik 2:15-28

Götz KG (1964) Optomotorische Untersuchungen des visuellen Systems einiger Augenmutanten der Fruchtfliege Drosophila. Kybernetik 2:77-92 
Hassenstein B, Reichardt W (1956) Systemtheoretische Analyse der Zeit-, Reihenfolgen- und Vorzeichenauswertung bei der Bewegungsperzeption des Rüsselkäfers Chlorophanus. Z Naturforsch 11b:513-524

Hausen K (1984) The lobula-complex of the fly: structure, function and significance in visual behaviour. In: Ali MA (ed) Photoreception and vision in invertebrates. Plenum Press, New York London, pp 523-559

Holub RA, Morton-Gibson M (1981) Response of visual cortical neurons of the cat to moving sinusoidal gratings: Responsecontrast functions and spatiotemporal interactions. J Neurophysiol 46:1244-1259

Kirschfeld K (1972) The visual system of Musca: studies on optics, structure and function. In: Wehner R (ed) Information processing in the visual systems of arthropods. Springer, Berlin Heidelberg New York, pp 61-74

Lappin JS, Bell HH (1976) The detection of coherence in moving random-dot patterns. Vision Res 16:161-168

Miles FA, Kawano K, Optican LM (1986) Short-latency ocular following responses of monkey. I. Dependence of temporospatial properties of visual input. $J$ Neurophysiol 56:1321-1354

Mimura K (1972) Neural mechanisms, subserving directional selectivity of movement in the optic lobe of the fly. J Comp Physiol 80:409-437

Pantle A, Lehmkuhle S, Candill M (1978) On the capacity of directionally selective mechanisms to encode different dimensions of moving stimuli. Perception 7:261-267

Pick B (1976) Visual pattern discrimination as an element of the fly's orientation behaviour. Biol Cybern 23:171-180

Poggio T, Reichardt W (1976) Visual control of orientation behaviour in the fly. Part II. Towards the underlying neural interactions. Q Rev Biophys 9:377-438

Reichardt W (1957) Autokorrelations-Auswertung als Funktionsprinzip des Zentralnervensystems (bei der optischen Wahrnehmung eines Insektes). Z Naturforsch 12b:448-457

Reichardt W (1961) Autocorrelation, a principle for evaluation of sensory information by the central nervous system. In: Rosenblith WA (ed) Principles of sensory communication. Wiley, New York, pp 303-317

Reichardt W (1986) Processing of optical information by the visual system of the fly. Vision Res 26:113-126
Reichardt W (1987) Evaluation of optical motion information by movement detectors. J Comp Physiol 161:533-547

Reichardt W, Guo A (1986) Elementary pattern discrimination (behavioural experiments with the fly Musca domestica). Biol Cybern 53:285-306

Reichardt W, Varjú D (1959) Übertragungseigenschaften im Auswertesystem für das Bewegungsehen (Folgerungen aus Experimenten an dem Rüsselkäfer Chlorophanus viridis). Z Naturforsch 14b:674-689

Reichardt W, Poggio T, Hausen K (1983) Figure-ground discrimination by relative movement in the visual system of the fly. Part II. Towards the neural circuitry. Biol Cybern [Suppl] 46:1-30

Riehle A, Franceschini N (1984) Research note. Motion detection in flies: parametric control over ON-OFF pathways. Exp Brain Res 54:390-394

Santen JPH van, Sperling G (1984) Temporal covariance model of human motion perception. J Opt Soc Am A 1:451-473

Santen JPH van, Sperling G (1985) Elaborated Reichardt detectors. J Opt Am A 2:300-321

Varjú D (1959) Optomotorische Reaktionen auf die Bewegung periodischer Helligkeitsmuster (Anwendung der Systemtheorie auf Experimente am Rüsselkäfer Chlorophanus viridis). Z Naturforsch 14b:724-735

Wehrhahn C (1986) Motion sensitive yaw torque responses of the housefly Musca: a quantitative study. Biol Cybern 55:275-280

Wilson HR (1985) A model for direction selectivity in threshold motion perception. Biol Cybern 51:213-222

Zeki SM (1974) Functional organization of a visual area in the posterior bank of the superior temporal sulcus of the monkey. J Physiol 236:549-573

Received: December 2, 1987

Prof. Dr. W. Reichardt

Max-Planck-Institut für biologische Kybernetik

Spemannstrasse 38

D-7400 Tübingen

Federal Republic of Germany 